\title{
« Il est interdit de... ». Rituels et procédures de régulation sensorielle dans le monde grec ancien : quelques pistes de réflexion
}

"One Shall Not... ». Regulating the Senses in the Ancient Greek Rituals: Some

Reflections

\section{Adeline Grand-Clément}

\section{OpenEdition}

\section{Journals}

Édition électronique

URL : http://journals.openedition.org/mythos/569

DOI : $10.4000 /$ mythos.569

ISSN : 2037-7746

\section{Éditeur}

Salvatore Sciascia Editore

\section{Édition imprimée}

Date de publication : 1 décembre 2017

Pagination : 49-58

ISSN : 1972-2516

Référence électronique

Adeline Grand-Clément, « « Il est interdit de... ». Rituels et procédures de régulation sensorielle dans le monde grec ancien : quelques pistes de réflexion », Mythos [En ligne], 11 | 2017, mis en ligne le 24 septembre 2019, consulté le 28 septembre 2019. URL : http://journals.openedition.org/mythos/569 ; DOI : $10.4000 /$ mythos.569 


\section{" Il est interdit de... ". Rituels et procédures de régulation sensorielle dans le monde grec ancien : quelques pistes de réflexion}

\section{Adeline Grand-Clément}

\section{Résumé}

II n'existait pas dans le monde grec de règlements religieux prescriptifs valables pour l'ensemble des lieux de culte et des rituels qui s'y déroulaient. Les normes variaient en fonction des endroits, des divinités, voire des périodes de l'année. Parmi les documents qui nous renseignent sur le déroulement des fêtes religieuses et l'attitude à adopter en pénétrant dans l'espace du sanctuaire, nous disposons d'un riche corpus d'inscriptions, longtemps connues sous l'appellation de "lois sacrées ". Cette documentation épigraphique a surtout été étudiée jusqu'ici du point de vue des autorités dont elles émanaient. L'objectif de l'article est d'envisager les prescriptions rituelles sous l'angle des effets qu'elles avaient sur les membres de la communauté qui s'y soumettaient, afin de déterminer si l'un des enjeux de la norme rituelle consistait précisément à agir sur le corps et l'expérience sensible des dévots. Après avoir évoqué la nature des interdits les plus fréquemment présents dans les règlements, l'étude se concentre sur les aspects sensoriels de quelques inscriptions associées au culte de Déméter dans le Péloponnèse, afin de déterminer ce qu'elles nous disent de la relation tissée entre les fidèles et la déesse.

\begin{abstract}
The Ancient Greek world didn't have rules that were applied in every shrines and for every rituals that were performed inside. The norms depended on the places, the deities, and sometimes the period of the year. Among the documentation which provides us with information on the way the religious festivals were performed and the behaviour expected on the part of the worshippers entering the sanctuaries, we find a rich series of inscriptions, commonly called "sacred laws". Until recently, these epigraphical datas have been studied from the point of view of the authorities which emitted the rules. The paper aims at shifting the perspective by studying the effects that such ritual prescriptions had upon the members of the community, in order to see to what extend the ritual norms were intented to act upon the body and the sensory experience of the worshippers. First, the paper sheds light upon the nature of the most common prohibitions to be found in the inscriptions. Then it focusses on the sensory effects produced by some rules connected to Demeter in the Peloponnesian, stating that these rules are meaningful regarding the relation established between the goddess and her worshippers.
\end{abstract}

\section{Mots clefs}

Normes rituelles • Déméter • Despoina • sensorialité • interdits • inscriptions

\section{Keywords}

Ritual norms • Demeter • Despoina • sensoriality • sensorium • prohibitions • inscriptions

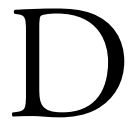
ans le système polythéiste grec, les dieux sont présents partout, au quotidien, et interfèrent dans le cours des vies humaines, même si on ne les voit pas ${ }^{1}$. Les rituels, pardelà les multiples formes qu'ils revêtent, visent à établir une communication entre les hommes et ces puissances invisibles. Pour avoir accès au monde supra-sensible, des dispositifs spécifiques sont mis en place de manière à solliciter le corps des participants, en instaurant une configuration sensorielle extra-ordinaire. Trois principaux types de stratégies peuvent être

1 Ils peuvent toutefois décider de se manifester, sous des formes épiphaniques qui ne mobilisent d'ailleurs pas uniquement le registre visuel : voir Piettre 2001 ; Platt 2011. 
employés : l'association intime de différents canaux sensoriels en même temps (poly-sensorialité), l'exacerbation de certains (hyper-sensorialité/saturation sensorielle) ou au contraire la privation d'un ou plusieurs type(s) de stimulus (hypo-sensorialité). Quelle que soit la stratégie mise en œuvre, elle fait intervenir plusieurs éléments déterminants. En premier lieu, le choix du lieu, l'emplacement du sanctuaire et sa configuration spatiale jouaient un rôle crucial. Le temenos (littéralement "espace séparé, coupé ") était considéré par les Grecs comme la propriété des dieux. Il y régnait une atmosphère singulière, signe de la présence du divin et fruit des interactions entre les propriétés intrinsèques du milieu naturel et divers éléments d'anthropisation : aménagements et constructions, mise en culture, plantations ${ }^{2} \ldots$ En deuxième lieu, les objets et les substances manipulés, voire ingérés - huile, vin, miel, encens, laine, eau, végétaux, sang - possédaient tous des qualités sensibles spécifiques, qui agissaient sur les participants en fonction de leur degré d'implication dans le rituel ${ }^{3}$. Enfin, les postures, les gestes, les mouvements et déplacements des corps des dévots, qui obéissaient à une séquence bien définie, généralement fixée par la tradition des ancêtres (ta patria), concouraient au caractère extraordinaire de l'expérience vécue lors des opérations rituelles. C'est notamment sur ce dernier point que nous allons nous attarder ici.

Parmi les documents qui nous renseignent sur le déroulement des fêtes religieuses et l'attitude à adopter en pénétrant dans l'espace du sanctuaire, nous disposons d'un riche corpus d'inscriptions, longtemps connues sous l'appellation de "lois sacrées ", qui couvrent un arc chronologique très large : de la période archaïque jusqu'à l'époque romaine. Il s'agit de normes rituelles émanant d'autorités diverses (association religieuse, cité, personnel du culte, roi...), qui étaient affichées sur des pierres ou des plaques en métal placées bien en vue à l'entrée des lieux de culte. De telles inscriptions ne livrent que des informations partielles, puisque les normes relevaient aussi de l'oralité. Aussi, il est malaisé de distinguer ce qui, dans cette documentation, relève de l'exception, d'un côté, et ce qui est commun et évident pour tous, de l'autre. Les opérations rituelles sont évoquées de façon elliptique, à travers les ingrédients nécessaires à leur accomplissement ${ }^{5}$. En dépit de ces limites, les inscriptions nous offrent un panorama très riche de la diversité des pratiques et donc des expériences religieuses grecques.

Cette documentation épigraphique a surtout été étudiée du point de vue des autorités dont elles émanaient. Les hellénistes se sont interrogés sur les raisons qui sous-tendaient le choix des prescriptions et des interdictions. Ils ont montré que le principal objectif était de garantir l'eukosmia, le "bon ordre ", la solidarité entre les participants du culte, et la pureté (hagneia), en luttant contre toute source de miasma, « souillure, pollution ${ }^{6}$. Mais cette documentation témoigne aussi d'un effort de manipulation du régime sensoriel ordinaire de la part des autorités en charge des opérations rituelles. Voilà pourquoi je suggère de considérer les règlements religieux sous un angle légèrement différent, en prêtant davantage attention aux effets qu'ils

2 Voir Brulé 2012.

3 Sur l'efficacité de ces éléments, en relation avec leur couleur, voir GRAND-CLÉmENT 2016a.

4 Une bonne partie de ce corpus a été édité par F. Sokolowski (LSAM;LSS;LSCG), et complété récemment par E. Lupu (NGSL). Une nouvelle édition du corpus épigraphique, au périmètre redéfini selon de nouveaux critères, a été initiée par Jan-Mathieu Carbon et Vinciane Pirenne-Delforge, qui s'expliquent sur leur démarche dans Carbon et Pirenne-Delforge 2012. Un premier état de ce "Corpus des Normes rituelles grecques" $(C G R N)$, destiné à être enrichi, est désormais accessible sur internet : http://cgrn.ulg.ac.be/.

5 Cf. Chaniotis 2010, 376.

6 Sur cette question de la pureté et de la souillure, voir l'étude de référence de PARKER 1983. 
sont susceptibles d'exercer sur le corps des dévots. En quoi les prescriptions rituelles contribuaient-elles à modifier les perceptions sensorielles de chaque participant(e) et agissaient-elles sur les interactions qui le/la liaient aux membres de la communauté ?

Je me bornerai à proposer quelques pistes de réflexion, en vue d'une réflexion plus large, en m'intéressant prioritairement à quelques cas d'interdictions ${ }^{7}$. Les travaux des anthropologues ont montré que les interdits façonnent la vision du monde des personnes qui y sont soumises et concourent à tisser un réseau de significations et de valeurs symboliques ${ }^{8}$. De plus, l'interdiction, parce qu'elle contribue à délimiter l'espace et le temps du rituel, nous renseigne sur le caractère " extra-ordinaire " de l'expérience vécue ${ }^{9}$. Nous commencerons donc par évoquer la nature des interdits les plus fréquemment présents dans les règlements, en essayant de prendre le compte le point de vue des dévots, pour saisir dans quelle mesure ils pouvaient s'en trouver affectés, jusque dans leur corps. Nous concentrerons ensuite notre attention sur les aspects sensoriels de quelques inscriptions associées au culte de Déméter dans le Péloponnèse, afin de déterminer ce qu'elles nous apprennent de la relation tissée entre les fidèles et la déesse.

\section{Les interdits : réguler les rituels et les comportements à l'intérieur du sanctuaire}

En pénétrant dans le temenos et en franchissant la frontière matérialisée par une porte ou des bornes, la/le dévot(e) acceptait de se soumettre à des règles spécifiques. On jugeait parfois bon de rappeler ces dernières, par exemple en les gravant sur le mur ou sur la borne (voir fig. 1).

Osons un parallèle avec les panneaux d'information actuels placés à l'entrée de lieux touristiques comme les musées, les parcs naturels ou les églises : eux aussi avertissent le visiteur

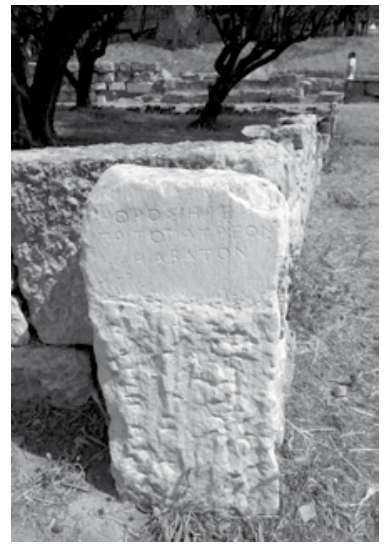

Fig. 1: Borne du sanctuaire des Tritopatores, Athènes, Céramique

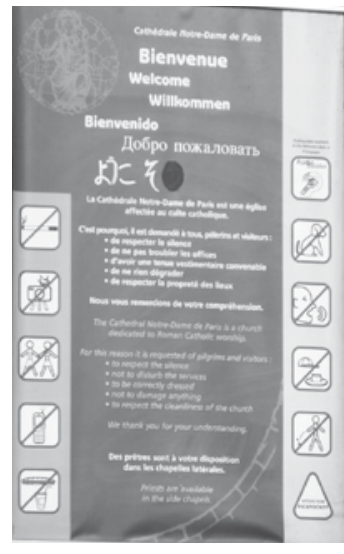

Fig. 2: Panneau placé à l'entrée de la Cathédrale NotreDame de Paris du type de règlementation qui y prévaut. Pour être aisément compris de tous, en particulier des étrangers, les textes sont assortis de pictogrammes. Ainsi, peut-on voir à l'entrée de Notre-Dame de Paris une quantité impressionnante de prescriptions qui fonctionnent toutes sur le mode de l'interdiction (fig. 2). On notera le caractère hétéroclite des interdictions énumérées : il s'agit à la fois de respecter le lieu de culte, de ne pas gêner les autres visiteurs et de garantir des conditions de sécurité. De fait, de tels panneaux nous renseignent autant sur la nature du lieu et sa fréquentation que sur le comportement habituel de celles et ceux qui y pénètrent et qui, temporaire-

7 J'ai conscience du caractère artificiel de la distinction entre ce qui est prescrit et ce qui est proscrit : certaines inscriptions associent étroitement les deux aspects, comme les deux versants d'un seul et même dispositif normatif.

8 Voir par exemple l'étude de Pierre Smith, qui se fonde sur une analyse de la notion d'umuziro au Rwanda (SмiтH 1979).

9 Il me semble en effet que lorsque les autorités estiment nécessaires de formuler et d'afficher une interdiction, c'est souvent pour aller à l'encontre d'un comportement adopté par certains fidèles, ou qui leur était commun hors de l'espace du hieron. 
ment, consentent à renoncer à certaines de leurs habitudes - en particulier vestimentaires. Dans le cas de Notre-Dame, toutefois, le non-respect d'une règle ne relève pas du domaine du sacrilège, à la différence de ce qui se passait dans un sanctuaire $\operatorname{grec}^{10}$. Autre différence : les inscriptions grecques ne recouraient pas à des pictogrammes, mais privilégiaient des formulations elliptiques. L'interdiction était généralement édictée en usant de la négation mè ou ouk, précédant le verbe à l'impératif ou à l'infinitif ${ }^{11}$. Certaines inscriptions, très courtes, étaient faciles à déchiffrer (comme par exemple celle stipulant "Entrée interdite ${ }^{12}$ ), tandis que d'autres, plus longues, nécessitaient probablement d'être lues par le personnel religieux présents sur place. Dans certains cas, on réaffirmait des règles traditionnelles censées être connues de tous, car communes à la plupart des sanctuaires grecs ${ }^{13}$, tandis que dans d'autres cas on souhaitait avertir plus spécifiquement les pèlerins étrangers des spécificités du culte local concerné, pour qu'ils ne commettent pas d'erreur et accomplissent correctement les gestes prescrits localement par les "traditions ancestrales" (ta patria). Par exemple, à Thasos, au début du Ve siècle av. J.-C., sur l'autel en marbre consacré à Apollon Nymphagétès et aux nymphes (ainsi qu'aux Charites et peut-être à Artémis, à Hermès et à Hécate, représentés par les anaglyphes), on ne pouvait sacrifier ni brebis ni porcelet, ni chanter des péans ${ }^{14}$. L'interdiction de sacrifier une brebis - l'une des victimes animales les plus communes - est unique dans notre documentation actuelle ; mais surtout, on s'étonnera de constater qu'à Thasos, Apollon ne puisse pas être honoré avec le chant qui le caractérise habituellement ${ }^{15}$. On ignore la raison d'être d'une telle spécificité locale, qui ne devait pas manquer d'intriguer les étrangers ${ }^{16}$.

Parce qu'ils visaient la concision et la clarté, les règlements n'explicitaient pas la raison d'être des interdits promulgués ${ }^{17}$. Les interdits touchaient de nombreux domaines ${ }^{18}$. Un nombre significatif concernait ce que nous appellerions la protection de l'environnement et du paysage :

10 Sur la notion de sacrilège, voir PARKer 1983, 144-190. Dans les inscriptions grecques, la mention des sanctions auxquelles s'expose le contrevenant (confiscation d'un bien prohibé, amende, mise au ban de la société, châtiment corporel...), n'est pas systématique. Quand rien n'est précisé, on ignore si l'inscription avait une simple fonction hortative et si le contrevenant était simplement rappelé à l'ordre ou expulsé du lieu.

11 Ajoutons que l'on trouve parfois ou themis ou ouk hosios/ouk hosia. Voir par exemple LSCG 136, qui concerne la refondation du calendrier cultuel à Ialysos (Rhodes). Sur la difficulté à traduire le terme hosios, qui renvoie à la sphère de la piété, voir PeELs 2016.

12 Par exemple une borne trouvée à Chios : hieron, ouk esodos (LSCG 121). On connaît des cas où l'accès est interdit seulement aux femmes : par exemple à Élatée, sur une borne du sanctuaire des Anakes (CGRN 33).

13 Ainsi, à Héraklée du Pont, au IV s. av. J.-C., une pierre délimitant l'entrée du sanctuaire rappelait un interdit habituel, qui ne devait être ignoré de personne - mais qui n’était peut-être pas toujours respecté : «Borne du hieron; défense d'y enterrer les morts " (LSAM 83).

14 LSCG 114.

15 Sur le péan et Apollon, voir BÉLIs 2001. C’est précisément le chant qui accompagne la procession de la longue procession des Molpoi de Didyme : voir Georgoud 2001.

16 D'après F. Sokolowski, on ne chantait pas de péan à cause des Charites qui bénéficiaient, à Paros (d'où les cultes avaient été transplantés à Thasos), de cérémonies sans musique ni couronnes (LSCG, 208). L'explication est réfutée par R. Parker $(2004,62)$.

17 L'interdiction n'avait pas besoin d'être précédée ou suivie d'une justification : elle faisait autorité pour ellemême, surtout si elle s'appuyait sur une consultation oraculaire et attribuait l'origine de la décision aux dieux. Un événement majeur induisant la réorganisation des cultes ou un transfert d'autorité du sanctuaire pouvaient être à l'origine de l'affichage d'un nouveau règlement. Des considérations économiques entraient aussi en ligne de compte. Les amendes prévues pour punir les infractions, ainsi que les confiscations de biens prohibés, alimentaient le trésor du sanctuaire.

18 Pour un bon aperçu de l'éventail des règles liées à l'entrée dans un sanctuaire, voir NGSL, 13-40. 
cela allait de l'abattage des arbres à la pâture des animaux, du dépôt d'ordures aux atteintes à la pureté de l'eau. Ainsi, dans le sanctuaire athénien d'Apollon Érithaseos, à la fin du IVe siècle av. J.-C., on ne pouvait pas couper du bois ni même ramasser branchages et feuilles mortes. Dans l'Asclépéion de Kos, au milieu du III s. s. av. J.-C., il était défendu de jeter des gâteaux ou toute autre chose dans les fontaines : les offrandes aux Nymphes devaient être déposées uniquement sur les autels. L'objectif des autorités était de préserver le cadre naturel du site - qui, d'après certains mythes de fondation, avait été choisi par les dieux eux-mêmes - ou plus exactement de garantir l'intégrité et la sécurité du temenos, propriété des dieux. Les autorités tutélaires du sanctuaire veillaient en outre à s'assurer du monopole de l'exploitation de ressources comme le bois, l'eau ou les pâtures, qui constituait une source de revenus non négligeable.

Tentons à présent d'adopter le point de vue des fidèles. De tels règlements les contraignaient à adapter leur comportement et à proscrire des gestes qui leur étaient familiers au quotidien. Ils avaient de fait le sentiment de pénétrer dans une sorte de conservatoire ou de "réserve naturelle ", marquée par une prédominance de bruits issus du règne animal ou végétal : sources, bruit du vent dans les arbres, chant des oiseaux. Les sources littéraires insistent d'ailleurs sur l'importance de la part naturelle du sanctuaire : c'est à cela que l'on reconnaît un lieu sacré19.

Mais il y a plus. La réceptivité du dévot aux stimulations extérieures se trouvait accrue par les exigences pesant sur son propre corps. Certains règlements établissaient en effet une liste détaillée d'interdits spécifiques, participant d'une recherche de sobriété et de mesure, voire d'une forme de dépouillement. D’autres se bornaient à exiger des dévots un état de pureté (hagneia), qui pouvait se traduire par la prescription de vêtements blancs ${ }^{20}$. Il s'agissait alors de marquer une différence avec le costume porté au quotidien. Les étoffes blanches exigeaient en effet un soin particulier pour conserver leur éclat et leur propreté ; nul doute qu'ainsi vêtus, les fidèles se comportaient de façon différente, adaptant leurs mouvements et leurs postures, parce qu'ils avaient conscience d'être "endimanchés".

La régulation en matière vestimentaire - que ce soit lors des funérailles, pour l'entrée dans un sanctuaire ou la participation à des fêtes, des processions et des cérémonies d'initiation - est justement l'aspect des « lois sacrées " qui a été le plus étudié par les chercheurs ${ }^{21}$. Le plus long texte dont on dispose concerne le déroulement des Mystères d'Andanie et date du début du $\mathrm{I}^{\mathrm{er}}$ siècle av. J.-C. On a en effet conservé une inscription qui détaille l'organisation de ces fêtes, prises en charge par la cité de Messène, dans le Péloponnèse. Les rédacteurs du règlement ont pris soin de distinguer les différents statuts des participants (personnel cultuel, simple initié, homme ou femme) en contrôlant scrupuleusement l'apparence vestimentaire de chacun, au sein de la procession ${ }^{22}$.

19 On citera la description effectuée par Antigone pour son père aveugle, dans le bois sacré des Euménides, à Colonne, au début de la pièce de Sophocle (Sophocle, Edipe à Colone, 16-20). Voir le commentaire de Brulé 2012, 39-41. Néanmoins, nous ne devons pas nous laisser leurrer par les termes employés, comme le «bois sacré», alsos, ou la "grotte ", antron : ils ne désignent pas nécessairement des espaces vierges de toute construction, bien au contraire ; le naturel faisait l'objet d'une ingénieuse mise en scène. Parfois antron désigne non pas une grotte naturelle, mais un local souterrain $(L S C G, 15)$.

20 Voir par exemple le règlement de Priène, daté du $\mathrm{II}^{\mathrm{e}}$ s. av. J.-C. : « Entrer dans le sanctuaire en étant pur, dans un vêtement blanc" (CGRN 121).

21 Sur les réglementations relatives au domaine vestimentaire, voir Milds 1984 ; Culham 1986, 235245, ou encore KaraTAS 2013.

22 Deshours 2006 ; GaWlinski 2012. 
La nécessité de réglementer le costume porté par les dévots, lors des fêtes, résultait d'un mélange de préoccupations différentes : des tabous anciens liés à la pureté rituelle ; des mesures somptuaires visant les aristocrates ; le souci de ne pas offenser la divinité ni de concurrencer le personnel religieux, seul autorisé à arborer certains vêtements distinctifs. Les différents paramètres jouaient un rôle plus ou moins déterminant en fonction des contextes et des traditions locales. On décèle toutefois une constante : c'étaient majoritairement sur les femmes que pesaient les restrictions relatives à l'apparence corporelle (coiffure, vêtement, bijoux...). Les règlements traduisaient en cela la volonté des autorités masculines de contrôler plus spécifiquement le comportement féminin (à travers leur costume), et donc leur visibilité dans l'espace public ${ }^{23}$.

Est-il possible de mesurer les effets - sur le plan émotionnel et sensoriel - que les mesures restrictives exerçaient sur les femmes et les hommes, lors de leur participation aux fêtes ou de leur visite dans le sanctuaire ? Trois inscriptions provenant du Péloponnèse (Achaïe et Arcadie), qui concernent le culte de Déméter ou d'une divinité associée (sa fille Despoina), nous serviront de matériau d'analyse. La première date de la fin de l'époque archaïque, les deux autres du III's. av. J.-C. - mais il n'est pas exclu qu'elles reproduisent en partie des interdits plus anciens. Les hellénistes ont pris l'habitude de les regrouper, parce que les interdits qu'elles contiennent procèdent d'une même exigence de pureté et de modération, voire d'une recherche d'un certain " dépouillement rituel " ${ }^{24}$. C'est ce qu'il faudrait examiner. Les interdits énoncés dans les trois inscriptions sélectionnées nous apprennent-ils quelque chose sur la façon dont les femmes éprouvaient les rites en rapport avec Déméter?

\section{Cultes de Déméter dans le Péloponnèse et "dépouillement rituel "}

La première, qui est aussi l'une des plus anciennes normes rituelles parvenues jusqu'à nous, provient du nord de l'Arcadie et concerne un sanctuaire dédié à Déméter Thesmophore. On la date de la fin du VIe siècle av. J.-C. Elle était gravée sur une petite plaque de bronze d'environ $25 \mathrm{~cm}$ de largeur et $10 \mathrm{~cm}$ de hauteur, probablement fixée sur une paroi, de façon à être lue. Le contexte de découverte est malheureusement inconnu : l'objet a été acquis sur le marché ${ }^{25}$. L.H. Jeffery a déduit de l'analyse paléographique et linguistique que l'inscription provient de la frontière avec l'Achaïe. Il suggère de la rattacher au sanctuaire de Déméter Thesmia, fondé par les citoyens de Phéneios, selon Pausanias (VIII, 15, 4) ${ }^{26}$. Le texte de l'inscription, relativement court, mentionne un magistrat, le demiourgos, ce qui laisse penser que la règlementation émane de la cité dont dépendait le sanctuaire. Il n'est pas exclu que cette plaque de métal ait été affichée avec d'autres, et fisse donc partie d'un ensemble de règles plus large concernant le sanctuaire :

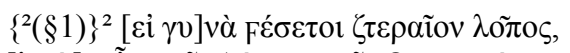

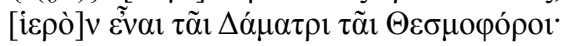

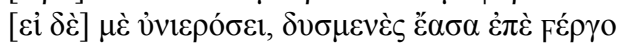

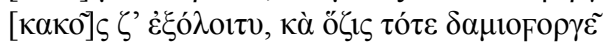

23 Voir Bielman 2002, 249 ; voir aussi sur ce point Cleland 2017.

24 Le Guen-Pollet 1991, 82-83 ; voir aussi Jost 1985, 330.

25 On trouvera la première édition commentée dans Robinson 1943.

26 JefFery 1949, 30-31. Madeleine Jost récuse toutefois cette interprétation (1985, 326). Le marchand qui a vendu la tablette disait qu'elle provenait de Kalavryta, de Lykosoura ou de Kleitor (cf. Pausanias, VIII, 21, 3) : voir RoBinson 1943, 191 (qui penche en faveur de la dernière provenance). 


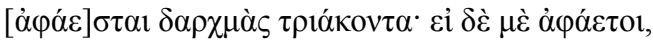

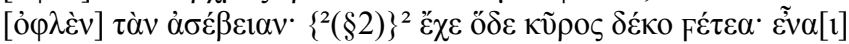

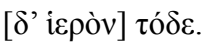

Si une femme porte un manteau décoré (?), qu'il soit consacré à Déméter Thesmophore ; si elle ne le consacre pas qu'elle soit bannie pour avoir été irrespectueuse envers la cérémonie (?), et que celui qui est alors démiurge fasse payer trente drachmes ; s'il ne fait pas payer, qu'il soit condamné pour impiété ; que cela soit ratifié pour dix ans ; que cette inscription soit sacrée ${ }^{27}$.

L'inscription concerne le culte de Déméter Thesmophore. Il s'agit de la plus ancienne attestation connue de l'épiclèse, sous laquelle la déesse était vénérée dans de nombreuses cités, notamment en Grande Grèce et à Athènes. Dans l'Hymne à Déméter composé par Callimaque au III ${ }^{\mathrm{e}}$ s. av. J.-C., le poète présente la déesse comme " celle qui a apporté les lois justes en même temps que les céréales et l'art de les cultiver " ${ }^{28}$. L'épiclèse Thesmophore souligne en effet le rôle joué par Déméter dans l'invention de la céréaliculture et l'instauration de mystères ${ }^{29}$.

L'interdiction concernait-elle de façon générale l'entrée dans le sanctuaire, ou seulement les périodes durant lesquelles étaient accomplis les rituels ? La seconde option est envisageable, si toutefois le terme ergon désigne bien une " cérémonie ", comme le suggère F. Sokolowski. La loi promulgue un seul interdit : celui de porter un certain type de vêtement, désigné par le syntagme zteraion lopos, difficile à traduire. Le terme lopos, rare, désigne vraisemblablement un manteau, porté sur les épaules ${ }^{30}$. La forme zteraion, quant à elle, est inconnue ailleurs. Il est possible de suggérer un rapprochement avec le terme zeira, qui désigne le long manteau bigarré des Arabes et des Thraces de l'armée immense de Xerxès, chez Hérodote (VII, 69 et 75). A. J. Beattie propose de traduire zteiraon par "brightly coloured ", " ornate ", " emboidered ", suggérant qu'il s'agit d'un équivalent de poikilos, "diapré, bariolé " ${ }^{31}$. L'interdiction d'un vêtement bigarré n'est pas unique dans la documentation épigraphique ${ }^{32}$. Le port de costumes très colorés - donc luxueux et coûteux - lors de réunions publiques faisait en effet l'objet d'un contrôle, pour éviter l'ostentation des aristocrates ${ }^{33}$ : des lois somptuaires promulguées dans les cités depuis la fin de l'époque archaïque visaient à limiter le poids des élites au bénéfice des autorités civiques.

Pour autant, il ne s'agit pas simplement d'une loi somptuaire ${ }^{34}$; rien d'autre n'est dit du reste de l'accoutrement, en particulier des bijoux. De plus, l'inscription souligne explicitement que l'interdit met en jeu la pureté rituelle : la transgression relève de l'asebeia (impiété) et menace l'intégrité du lieu sacré. Il est précisé que le vêtement de la contrevenante devait être offert à la déesse. Une telle mesure " réparatrice " n'a rien d'étonnant, quand on sait que les tissus

27 LSS 32 ; traduction de Marie Augier, légèrement modifiée.

28 Callimaque, Hymne à Déméter, 18-20.

29 STALlsmith 2008.

30 Voir Odyssée, XIII, 224 ; il s'agit d'un terme plutôt employé par les poètes, selon le LSJ (s.v. lopè).

31 Beattie 1947, 67-68. L'auteur rejette la suggestion de Robinson qui, en vertu d'un rapprochement avec deiraion, dermatikon, estime qu'il s'agit d'un manteau de cuir (RoBInson 1943, 194).

32 Voir par exemple des cas analogues à Kios et à Délos (c'est alors l'adjectif anthinon, «fleuri, aux couleurs vives " qui est utilisé) : LSS 28.

33 Grand-Clément 2011, 269-271.

34 C'est l'interprétation qu'en donne par exemple Jost 1985, 326. 
faisaient partie des consécrations les plus fréquentes dans certains sanctuaires ; il s'agissait de pièces de valeur réalisées à dessein par les femmes, en signe de piétéc ${ }^{35}$. On sait d'ailleurs, grâce à une inscription fragmentaire relative au culte de Déméter à Sparte, qui date du VI ${ }^{\mathrm{e}}$ siècle av. J.C., que l'offrande d'étoffes tissées par les femmes s'effectuait sous le contrôle des magistrats ${ }^{36}$.

Cela nous amène à formuler une autre remarque : seules les femmes étaient concernées. Le terme gunè étant générique, il est impossible de déterminer s'il renvoyait uniquement aux épouses de citoyens, ou s'il incluait toute la composante féminine de la cité (c'est-à-dire les étrangères, les esclaves, les jeunes filles...). Quoi qu'il en soit, les hommes, eux, n'étaient pas visés. Deux explications sont possibles : soit les hommes ne faisaient pas l'objet d'une régulation vestimentaire (ou ne portaient pas de manteau bariolé), soit le culte de Déméter Thesmophore - voire même l'accès à son sanctuaire ${ }^{37}$ - était exclusivement réservé aux femmes. Rappelons que, dans des cités comme Athènes, l'organisation des Thesmophories, qui visaient à garantir la reproduction du corps civique et la fécondité des champs, était prise en charge par les épouses des citoyens; les hommes n'y prenaient pas part ${ }^{38}$. Les fêtes commençaient par une période de jeûne au cours duquel les femmes expérimentaient la souffrance de Déméter séparée de sa fille et elles se terminaient par un jour de liesse, célébrant les retrouvailles des deux déesses. Hérodote, qui en parle comme des "fêtes d'initiation » (teletès) d'origine égyptienne, précise que la tradition avait été conservée principalement en $\operatorname{Arcadie}^{39}$ : le fait que cette inscription en provienne et qu'elle nous fournisse justement la plus ancienne attestation de l'épiclèse Thesmophore appliquée à Déméter semble appuyer le témoignage de l'historien.

La deuxième inscription qui nous intéresse a été trouvée dans la région voisine d'Achaïe, près de la frontière avec l'Élide. Le texte était gravé sur une plaque rectangulaire en pierre grise, découverte dans un sanctuaire localisé dans un vallon, près de l'actuel village de Petrochôrion, sur la côte au sud-ouest de Patras. Ce lieu de culte extra-urbain appartenait au territoire de la cité de Dymé. L'inscription est relativement bien conservée ; seule la partie haute de la pierre est cassée ${ }^{40}$. Le règlement, nettement postérieur au précédent, puisqu'on le date de la fin du $\mathrm{III}^{\mathrm{e}}$ siècle av. J.-C., concerne également un culte de Déméter. Il présente des ressemblances frappantes, même si la palette d'interdits est plus développée :

$$
\begin{aligned}
& {\left[.{ }^{?} \cdot .\right] \Delta[\alpha]-} \\
& \mu \alpha \tau \rho i ́ o ı \varsigma \tau \grave{\alpha} \varsigma \gamma v v[\alpha \tilde{i}]-
\end{aligned}
$$

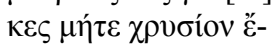

$$
\begin{aligned}
& \chi \varepsilon v \pi \lambda \varepsilon \dot{0} \text { ov ỏ } \delta \varepsilon \lambda \circ \text { õ ó } \lambda \text { - }
\end{aligned}
$$

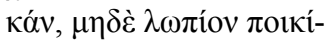

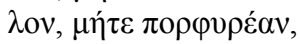

35 Sur les consécrations de tissus, voir notamment Morizot 2004 et NeILs 2009.

36 LSS 28.

37 Pausanias mentionne l'existence, près de Mégalopolis, en Arcadie, d'un sanctuaire de Déméter des marais, dont l'accès est réservé aux femmes : Périégèse, VIII, 36, 6.

38 Une inscription de Laconie mentionne des fêtes de Déméter interdites aux " mâles " : cf. LSCG 63.

39 Hérodote II, 171 : "De même sur les fêtes d'initiation de Déméter, que les Grecs appellent Thesmophories, sur ces fêtes aussi gardons le silence, sinon pour en dire ce que permet la piété : ce sont les filles de Danaos qui ont apporté ces rites d'Égypte et les ont enseignés aux femmes des Pélasges ; plus tard, quand la population du Péloponnèse fut contrainte d'émigrer par les Doriens, les rites en question se perdirent ; seuls, les ont conservés ceux des Péloponnésiens qui demeurèrent sur place et n'émigrèrent pas, les Arcadiens ».

40 Dimensions : 48x40x7-10 cm ; Musée de Patras, inv. 149 (pour une photographie, voir pl. II de RizAKIs 2008). 


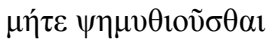

$\mu \eta \dot{\tau} \varepsilon \alpha \dot{0} \lambda \tilde{\eta} \nu \cdot \varepsilon \dot{~} \delta \varepsilon \dot{\varepsilon} \kappa \alpha$

$\pi \alpha \rho \beta \alpha ́ \lambda \lambda \eta \tau \alpha$, $\tau$ ò̀ i-

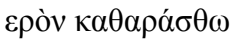

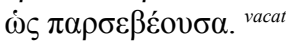

[...] Pour les fêtes en l'honneur de Déméter, que les femmes n'aient pas d'objet en or pesant plus d'une obole, ni de manteau diapré ou pourpre, qu'elles ne soient pas fardées de blanc et qu'elles ne jouent pas de flûte (aulos); en cas d'infraction, qu'elle purifie (fasse purifier ?) le sanctuaire en tant qu'impie ${ }^{41}$.

Les interdits sont ici liés explicitement à la célébration de fêtes religieuses nommées Démétria. On ignore la façon dont elles se déroulaient, mais on peut envisager là encore des cérémonies de type Thesmophories (ce que fait d'ailleurs l'éditeur de l'inscription, Athanassios Rizakis) ${ }^{42}$. Le texte rattache explicitement l'interdit à la sphère de l'eusebeia (piété), puisqu'une purification du sanctuaire était nécessaire en cas d'infraction ${ }^{43}$. Ici encore, le contrôle de l'apparence vestimentaire s'exerce sur les femmes. Les restrictions sont toutefois plus nombreuses et variées. Le premier interdit concerne, comme pour le culte arcadien de Déméter Thesmophore, le manteau (lopion) des participantes aux cérémonies : non seulement il ne doit pas être richement décoré (poikilos), mais l'inscription ajoute la proscription d'une couleur en particulier, à savoir la pourpre. Cela n'a rien d'étonnant. Il s'agit en effet de la couleur vestimentaire la plus régulée dans les inscriptions relatives aux rituels, à partir de l'époque hellénistique : la pourpre était tantôt proscrite, pour les participants aux rituels, tantôt prescrite pour les membres du personnel religieux et la parure des statues divines. Le prestige considérable dont bénéficiait cette teinture dans le monde grec tenait à sa valeur économique mais aussi à la force des résonances symboliques qui s'y rattachaient : il s'agissait d'une couleur d'apparat arborée par les puissants, et qui évoquaient l'immortalitéét. En interdisant un manteau pourpre, la loi de Dymé garantissait une forme de modération dans l'accoutrement des femmes issues des couches sociales les plus élevées.

La restriction concernant le poids des bijoux en or autorisés rejoint sans doute de telles préoccupations. Comme la pourpre, l'or occupait une place particulière dans l'imaginaire grec : ce métal à l'éclat inaltérable était intimement associé aux dieux, et de nombreux objets servant pour le culte étaient fabriqués dans cette matière brillante aux reflets solaires ${ }^{45}$. Si les vêtements des fidèles étaient souvent blancs, ceux des prêtres arboraient des couleurs distinctives, en particulier l'or, la pourpre ou l'écarlate ${ }^{46}$. Il ne s'agissait pas juste de signaler visuellement l'existence d'une hiérarchie : à la fonction sémiotique s'ajoutait une fonction symbolique et performative, dans la mesure où l'or et la pourpre étaient deux substances supposées activer la communication avec les dieux ${ }^{47}$.

41 Rizakis 2008, n6 = LSS, n³3 A = CGRN 127 (trad. J. Carbon modifiée).

42 Malheureusement, nos sources tant archéologiques, épigraphiques que littéraires ne livrent aucune information concernant le culte de Déméter à Dymé.

43 Voir Delli Pizzi 2011.

44 Grand-Clément 2016 b.

45 Sur la fonction de l'or dans le culte, avec même des vertus purificatoires qui lui étaient attribuées, voir GEORGOUDI 2014

46 JONES 1999.

47 Grand-Clément 2016 b. 
On apprend en outre que le maquillage était également proscrit lors des fêtes de Déméter. Le mot psimuthion désigne le blanc de plomb ou céruse, un produit utilisé par les femmes grecques comme fond de teint. Nous avons là affaire à une mesure exceptionnelle : la seule autre attestation épigraphique connue à ce jour dans le corpus des normes rituelles grecques concerne les participantes aux Mystères d'Andanie, dont il a été question plus haut ${ }^{48}$. La pratique de se "blanchir " le visage, pour avoir un teint clair et lumineux en se conformant ainsi à l'idéal de beauté aristocratique pour les femmes, est attestée par les sources littéraires mais aussi archéologiques, depuis l'époque archaïque. Certains auteurs grecs d'époque classique, comme Xénophon ou Platon, condamnent le maquillage : de tels artifices masquent la beauté véritable et trompent l'entourage ${ }^{49}$. Les fards n'étaient pas de mise pour les femmes portant le deuil $^{50}$. De telles considérations ont joué dans la promulgation de l'interdiction du psimuthion à Dymé. Se présenter sans fard devant la divinité permettait d'afficher un état de pureté garantissant le succès de l'opération rituelle; plus que jamais simplicité et aidôs étaient de rigueur ${ }^{51}$. Les autorités civiques avaient à cœur de contrôler étroitement le comportement des femmes lorsqu'elles prenaient en charge les rituels cruciaux pour la survie de la communauté, ce qui était le cas avec le culte de Déméter ${ }^{52}$. La célébration de fêtes comme les Thesmophories était d'ailleurs marquée par une période d'abstinence sexuelle. La loi de Dymé contribuait de fait à dépouiller les femmes de tous les attributs symbolisant la séduction et l'attraction sexuelle $e^{53}$.

Une autre spécificité des Démétria concerne l'absence d'aulos, qui est plutôt inhabituelle. En effet, cet instrument de musique, ancêtre du hautbois, était abondamment utilisé dans le monde grec. L'aulos servait à marquer la cadence des pas et jouait, comme dans le monde romain, le rôle de paravent acoustique au moment de mettre à mort l'animal. De nombreuses inscriptions signalent ainsi la présence d'un aulète aux côtés du héraut, lors des rituels. L'aulos fournissait donc la trame sonore habituelle des processions et des sacrifices dans le monde grec ${ }^{54}$. Là encore, l'interdit est rarement attesté ailleurs ; on connaît le cas du culte de Dionysos Brômios à Smyrne ${ }^{55}$. L'interdiction de jouer de la flûte dans l'un et l'autre cas visait à empêcher ce qui, au regard du rite concerné, pouvait être perçu comme une forme de nuisance, de perturbation. Avec Dionysos Brômios, le "Grondant ", il s'agissait peut-être de laisser à la divinité le monopole de la sphère acoustique. Quoi qu'il en soit, l'interdit avait pour effet de colorer le lieu et la célébration concer-

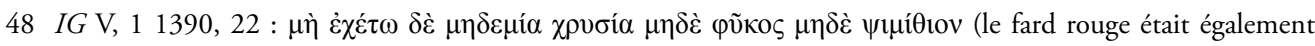
proscrit).

49 Xénophon, Économique, X ; sur le maquillage, voir GHerchanoc 2011.

50 Lysias, Sur le meurtre d'Eratosthène, 14.

51 GHERCHaNoc 2011.

52 PArker 1983, 82-83 : "The ritual focuses attention on the idea of productive sexual union by a paradoxical temporary insistence upon its opposite ". L'auteur rappelle à juste titre qu'il n'y avait rien de proprement impur dans les objets eux-mêmes : " it is polluting in this context because it offends against the ethos of a festival that requires women temporarily to renounce the paraphernalia of sexual attraction" (145).

53 Les premières visées étaient peut-être les courtisanes, dont le costume, très voyant, comprenait couleurs vives, tissus pourpres et bijoux en or, et qui se maquillaient ; voir PARker 1983, 83, n. 36. Phylarchos (III s. av. J.-C.), rapporte qu'une loi syracusaine remontant au VIe siècle av. J.-C. stipulait que le port d'une robe "fleurie ", de vêtements bordés de pourpre et de bijoux en or était le propre des hétaïres. Ajoutons que l'aulos était également associé au monde du banquet et aux divertissements - certaines courtisanes étaient expertes dans l'art de jouer de la flûte. Or l'usage de l'aulos est justement prohibé lors des Démétria.

54 Voir Piоцот 2001, qui souligne le rôle de l'aulos dans l'efficacité de l'opération rituelle.

55 Rizakis 2008, 62 relève un autre cas d'interdiction de flûte pour les sacrifices. 
née d'une signature sonore singulière, pour des oreilles grecques habituées au son de l'aulos.

Il ne faudrait pas exagérer la portée des interdits de Dymè et l'effet qu'ils avaient sur les personnes concernées : toutes les femmes grecques ne recouraient pas au maquillage et ne possédaient ni gros bijoux en or, ni vêtements pourpres ou richement décorés. Néanmoins, pour les plus riches, le fait de se dépouiller revenait à abandonner les signes distinctifs de leur appartenance à l'élite, pour se fondre dans le groupe. L'acceptation du règlement constituait en outre une marque de respect et le signe de reconnaissance d'une autorité supérieure : il fallait éviter à tout prix de contrarier Déméter. Les Grecques savaient que les colères de la déesse pouvaient être redoutables, lorsqu'elle se sentait offensée ${ }^{56}$. Il est donc probable que le dénuement relatif exigé des dévotes avait, de leur point de vue, plusieurs effets : éprouver un contact plus intime avec le divin ; accepter de reconnaître la toute puissance de Déméter, à laquelle chacune, quelle que soit son origine, devait se soumettre ; se sentir partie intégrante d'une même communauté, marquée par l'abandon des signes ostentatoires de sa propre identité sociale.

Les différentes interdictions énoncées dans l'inscription conféraient de surcroît une tonalité originale aux Démétria. En éliminant les sources potentielles de stimulation sensorielle, susceptibles de détourner l'attention des participantes (couleurs vives, éclats des matières, son pénétrant de l'aulos), la norme rituelle garantissait une atmosphère propice au recueillement. Leur réceptivité s'en trouvait accrue, leurs perceptions sensorielles aiguisées. L'impact émotionnel reste difficile à évaluer, mais il faut garder en mémoire que lors de certaines fêtes en l'honneur de Déméter, à l'instar des Thesmophories, nous l'avons dit, les femmes étaient amenées à partager temporairement les sentiments d'affliction éprouvés par la déesse en quête de sa fille, enlevée par Hadès. En renonçant à un certain nombre d'attributs de leur féminité, les dévotes agissaient comme Déméter, se soumettant à une forme de retraite temporaire. Les mythes mettant en scène la déesse courroucée étaient d'ailleurs nombreux, notamment dans le Péloponnèse ${ }^{57}$ : l'un d'entre eux nous amène jusqu'à Lykosoura, où régnait Despoina, la fille de Déméter, née du viol que Poséidon avait fait subir à cette dernière.

\section{Despoina à Lykosoura}

Lykosoura, situé non loin de Mégalopolis, était l'un des principaux (et le plus ancien) sanctuaires d'Arcadie. Il attirait des dévots de l'ensemble de la région. Pausanias, qui l'a visité au $\mathrm{II}^{\mathrm{e}}$ s. ap. J.-C., fournit un certain nombre de renseignements précieux à son sujet ${ }^{58}$. Le sanctuaire était consacré à une divinité chtonienne locale nommée Despoina (" la Souveraine »), qui était vénérée aux côtés de sa mère Déméter, ainsi que d'Artémis et du héros Anytos. Les fouilles effectuées sur le site ont mis au jour les principaux éléments d'organisation du temenos. Dans le temple, on a retrouvé des fragments des statues monumentales que le célèbre sculpteur Damophon y fit construire vers 300 av. J.-C. Ce groupe monumental en marbre, de 4 mètres de haut, faisait l'objet d'une mise en scène sophistiquée destinée à mettre en valeur l'épiphanie

56 Dans son Hymne à Déméter, Callimaque évoque la colère noire dans laquelle entre la déesse lorsque l'on s'en prend aux arbres de son bois sacré, qu'elle affectionne tant.

57 Non loin de là, à Phigalie, Déméter était vénérée dans une grotte, sous l'épiclèse de Melaina : c'est là qu'elle s'était retirée après le rapt de sa fille. Pour marquer sa douleur et son affliction, elle s'était drapée de noir, ce qui expliquait l'épiclèse sous laquelle elle était vénérée : Pausanias VIII, 42, 2-4. Finalement apaisée par les Moires, elle avait consenti à quitter la grotte et à abandonner son vêtement de deuil.

58 Pausanias, VIII, 37. 
divine ${ }^{59}$. Pausanias raconte qu'on célébrait pour Despoina et Déméter des " Mystères », qui commençaient par une procession regroupant les initiés et futurs initiés ${ }^{60}$. On sait malheureusement peu de choses du déroulement de ces rituels, mais les découvertes archéologiques et les témoignages iconographiques incitent à imaginer des danses, accompagnées de musique (dont l'aulos), par des personnages portant des masques d'animaux ${ }^{61}$. Madeleine Jost estime que la pénétration de l'influence éleusienne en Arcadie, à partir du Ve s. av. J.-C., n’a finalement touché les Mystères de Lykosoura que de façon superficielle : Déméter y jouait un rôle secondaire par rapport à Despoina, et même par rapport à Artémis ${ }^{62}$.

Deux inscriptions sur pierre, l'une de la fin du III ${ }^{e}$ siècle, l'autre du II ${ }^{e}$ siècle av. J.-C., nous renseignent sur les pratiques rituelles prescrites à l'intérieur du sanctuaire ${ }^{63}$. Arrêtons-nous sur la première, la plus développée, qui avait été gravée sur une stèle placée à l'entrée du sanctuaire :
$\Delta \varepsilon \sigma$ Toívas.
[...] Mì $\dot{\varepsilon} \xi \dot{\varepsilon} \sigma \tau \omega$

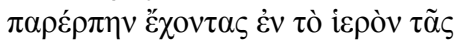

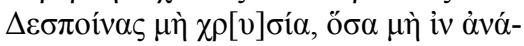

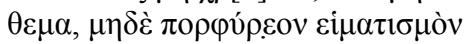

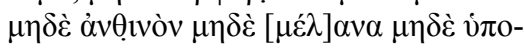

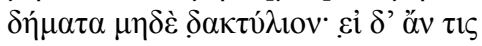

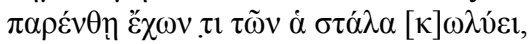

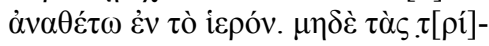
$\chi \alpha \varsigma \dot{\alpha} \mu \pi \varepsilon \pi \lambda \varepsilon \gamma \mu \varepsilon \dot{v} v \alpha \varsigma \mu \eta \delta \dot{\varepsilon} \kappa \varepsilon \kappa \alpha \lambda v \mu-$

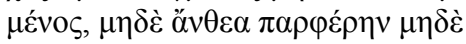

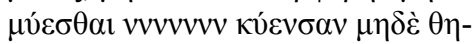

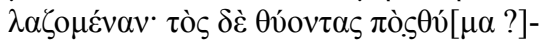

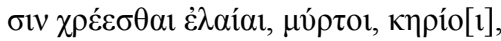

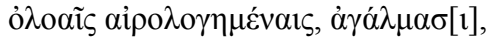

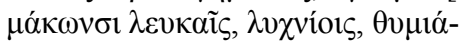

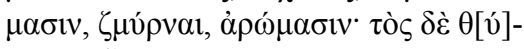

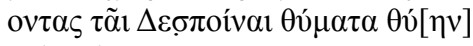

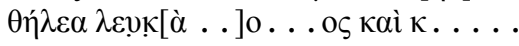

(Sanctuaire) de Despoina. [...] Défense de pénétrer dans le sanctuaire de Despoina avec des objets en or, sauf en vue de leur consécration, avec un vêtement teint de pourpre ou fleuri ou noir, avec des sandales, avec un anneau. Au cas où quelqu'un entrerait avec

59 Pausanias explique : «En sortant du temple vous verrez sur votre droite un miroir encastré dans le mur ; quand on s'y regarde à peine se voit-on, mais on voit distinctement ce beau groupe de marbre dont j'ai parlé. " Sur ce dispositif, voir Montel 2008.

60 Pausanias, VIII, 37, 2.

61 Voir Jost 1985, 333-334. L'auteur insiste sur l'aspect exceptionnel de ces rites (337) - ce qui pourrait justifier l'affichage d'une réglementation spécifique.

62 Voir Jost 1985, 353-355.

63 La première a été trouvée dans un champ, au sud de la ville : $I G \mathrm{~V}, 2,514$; elle est conservée au Musée épigraphique d'Athènes (inv. 11522). La seconde, plus fragmentaire (seulement 6 lignes lisibles), a été trouvée en réemploi dans une chapelle, mais était probablement elle aussi placée à l'entrée du sanctuaire : NGSL, 215-218, $\mathrm{n}^{\circ} 8$. Elle concernait les conditions de pureté nécessaires avant d'entrer et la nécessite de sacrifier conformément aux prescriptions du prêtre. Il est possible que cette seconde loi ait visé à renforcer les prescriptions de la première, dans un contexte de compétition avec la cité voisine de Mégalopolis, où venaient d'être introduits les Mystères éleusiniens. 
l'un des objets que la stèle proscrit, que celui-ci soit consacré dans le sanctuaire. Défense d'avoir les cheveux tressés ou la tête couverte ; défense d'apporter des fleurs, défense de se faire initier [vacat] pour une femme enceinte ou qui allaite. Que les personnes qui sacrifient utilisent en guise d'offrandes additionnelles de l'olivier et du myrte, des rayons de miel, des grains d'orge sans ivraie, des agalmata ${ }^{64}$, des pavots blancs et des lampes, des parfums à brûler, de la myrrhe et des aromates. Que ceux qui offrent des sacrifices à Despoina sacrifient des victimes femelles blanches ${ }^{65} \ldots$

L'inscription, lacunaire (il manque la fin), combine en fait plusieurs types de réglementation. Le début énonce des interdictions relatives au vêtement et aux parures des fidèles entrant dans le sanctuaire, avec confiscation prévue pour les contrevenants. Ici les femmes ne sont pas mentionnées explicitement, sauf en ce qui concerne les restrictions relatives à l'initiation. Il semblerait néanmoins que, comme précédemment, les interdits vestimentaires les concernaient prioritairement, car les objets incriminés relevaient bien de la panoplie féminine : vêtements colorés, parures pour les cheveux et bijoux en or. On trouve ensuite dans l'inscription des interdictions relatives aux futurs initiés, suivies des prescriptions relatives à la nature des offrandes à effectuer ${ }^{66}$. Le texte gravé sur la pierre agrégeait donc des règlements relatifs à des lieux et à des moments distincts. La nécessité de compiler ces instructions s'est sans doute fait sentir en raison d'un accroissement de la fréquentation du site, qui s'est traduit aussi, nous l'avons vu, par l'effort de monumentalisation perceptible à partir de 300 av. J.-C.

L'accumulation des interdits est significative : elle reflète l'ampleur de la fréquentation du sanctuaire et le caractère bigarré de la foule des pèlerins. Les autorités du sanctuaire ont cherché à faire face à tous les types de situation. Cela nous rappelle la longue inscription des Mystères d'Andanie. On y retrouve d'ailleurs un vocabulaire et des formulations analogues. Par exemple, à Andanie, le terme eimatismon y désigne de manière spécifique le costume prescrit pour les femmes, pour la " représentation des dieux ", qui fait l'objet d'un strict contrôle. Le diagramma stipulait en effet, au sujet de la procession : "Et que toutes celles qui doivent s'équiper pour la représentation des divinités portent le vêtement que les hieroi auront prescrit. Si quelqu'un porte son vêtement autrement, contrairement au règlement, ou autre chose d'interdit, que le gynéconome s'y oppose et qu'il lui soit permis d'arracher (l'objet) et qu'il soit consacré aux dieux ${ }^{67}$. "

Le texte de Lykosoura contient plusieurs nouveautés par rapport à ce que nous avons vu dans les deux autres inscriptions. Premièrement, l'interdiction du vêtement noir. Serait-ce simplement une façon de prescrire du blanc, comme on le lit parfois ${ }^{68}$ ? Certes, on connaît de nombreux exemples pour les époques hellénistique et romaine de vêtements blancs requis pour entrer dans un sanctuaire ou participer à une cérémonie ${ }^{69}$, mais il me semble alors

64 La traduction du terme est problématique. Voir Loucas, Loucas- Durie 1985-1986. Sur les différentes acceptions du terme, voir LANÉRÈs 2012.

$65 I G \mathrm{~V} 2$ 2, 514 ; trad. Madeleine Jost $(2008,94)$ modifiée. Voir aussi Loucas, Loucas 1994.

66 Nous ne nous attarderons pas ici sur ce dernier point.

$67 I G \mathrm{~V}(1), 1390,24-26$. Un peu plus bas (1. 36), à propos de l'enclos réservé aux hieroi où sont installées les tentes et dont le périmètre est délimité par des bandelettes, il est précisé que ceux-ci doivent inscrivent quels objets il

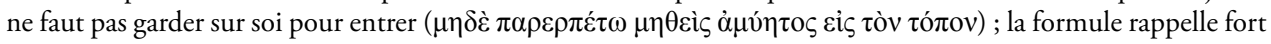
le début de notre inscription.

68 Jost 2008, 96.

69 Par exemple dans le sanctuaire d'Asclépios à Pergame ou à Délos, à l'entrée du sanctuaire d'Athéna Kinthia et Zeus Kinthios. Voir aussi Pausanias, VI, 20, 2-3. 
qu'il aurait suffi d'exiger explicitement du blanc, comme c'était stipulé dans le diagramma d'Andanie au sujet des initiés. La proscription du bigarré, du pourpre et du noir ne signifiait pas obligatoirement prescription du blanc : il restait toute une gamme de couleurs possibles. Il faut donc à mon avis s'interroger sur la raison qui explique un interdit spécifique de la couleur noire. Le seul autre cas connu, dans la documentation épigraphique, concerne le culte de Dionysos Brômios, à Smyrne, dont nous avons déjà parlé au sujet de l'aulos ${ }^{70}$. On sait que les Grecs associaient les vêtements noirs, ou plus généralement sombres, au deuil et aux funérailles ${ }^{71}$. On peut donc imaginer que l'interdit visait à exclure du sanctuaire les femmes portant le deuil, donc encore entachées de la souillure liées à la mort. On connaît en effet de nombreux règlements imposant aux femmes ayant été en contact avec un cadavre un certain délai avant de pouvoir de pénétrer dans un hieron ou de participer à une fête. Mais pourquoi l'évoquer de manière détournée ici, par le biais d'une proscription du noir ? Est-ce parce que l'exigence devenait impérieuse dans le cadre de ce culte ? L'interdit lié à la couleur noire n’avait-il pas à voir avec la déesse et sa sphère de compétence ? On constate en effet, grâce à la suite de l'inscription, que les offrandes destinées à Despoina étaient plutôt du côté de la blancheur que de la noirceur ${ }^{72}$.

Deuxième nouveauté : l'interdiction des sandales. Il s'agit là d'une mesure moins originale, qui n'est nullement réservée au culte de Déméter. On la retrouve dans d'autres inscriptions, formulée de deux façons : soit en prohibant les chaussures - parfois selon leur couleur ou la matière dans laquelle elles sont réalisées ${ }^{73}$; soit en exigeant explicitement des dévots qu'ils soient "pieds nus " (anupodetous) ${ }^{74}$. Il est difficile de déterminer si la variation de formulation est significative. Dans le cas qui nous intéresse, le choix de privilégier l'angle de l'interdiction est lié aux mesures précédentes : tous les accessoires prohibés venaient alimenter le trésor sacré. L'accumulation des interdits participait ainsi à donner la mesure de l'étendue de la puissance de Despoina et de Déméter. Parmi les raisons suggérées pour expliquer l'interdiction des chaussures, l'exigence de pureté revient souvent ${ }^{75}$. Mais examinons ce que cela faisait concrètement aux fidèles. Le fait de devoir se déchausser en entrant dans le sanctuaire renforçait en eux le sentiment de franchir un seuil - de passer du dehors au-dedans, de pénétrer comme un hôte dans le domaine de la divinité. Se déplaçant pieds nus, ils devaient

70 LSAM 84, 10. Cf. Pugliese Carratelli 1957, 444 (l'auteur estime que c'est la connotation funéraire des vêtements noirs qui est en jeu). Nous gagnerions à explorer davantage les similitudes constatées entre certains cultes à Déméter et à Dionysos; le dossier des rites mystériques fournit des données intéressantes, du point de vue de la sensorialité (Ustinova 2013).

71 Sur les inscriptions relatives aux rites funéraires, voir FrISONE 2000.

72 Ajoutons que Déméter elle-même, à Phigalie, avait abandonné son vêtement noir, lorsque les Moires l'avaient apaisée et persuadée de quitter la grotte dans laquelle elle s'était retirée : Pausanias VIII, 42, 2-4.

73 Par exemple à Ialysos (Rhodes), dans le sanctuaire d'Alektrôna, vers 300 av. J.-C., sont interdites les chaussures ou choses faites avec de la peau de cochon : LSCG $136=$ CGRN 90 .

74 C'est le cas dans différents contextes rituels : pour entrer dans un sanctuaire comme celui d'Athéna et de Zeus du Cynthe à Délos (l'inscription date du II $\mathrm{e}$ s. av. J.-C. mais elle reproduit sans doute une règle plus ancienne : LSS 59) ou celui d'Athéna Lindia, à Rhodes, au III' s. ap. J.-C. (il faut être anupodetous ou alors avoir des sandales blanches mais non faites de chèvre : LSS 91), lors d'une cérémonie (pour Déméter à Chios au I ${ }^{\text {er }}$ s. ou pour un sacrifice à Zeus Hypatos au II siècle av. J.-C., à Cyrène = LSS 116), pour passer une nuit dans le sanctuaire d'Asclépios à Pergame (III s. ap. J.-C., LSAM 14, 11), ou encore pour participer à une procession (on sait par exemple que les initiés (hommes et femmes) des Mystères d'Andanie doivent être pieds nus).

75 Ainsi Parker 1983, 177. 
sentir le sol, sa texture, sa température, et modifiaient en conséquence leur façon de marcher. Les archéologues estiment que les pavements en pierre se développèrent surtout à l'époque romaine ; jusque-là, les différentes voies menant des entrées des sanctuaires à l'autel n'étaient pas dallées : le contact avec la terre était donc direct ${ }^{76}$. C'est peut-être ce contact qui était recherché, comme une manière d'intensifier la communication avec le divin, ou du moins de l'amorcer. De plus, sans chaussure, la marche se faisait plus lente et précautionneuse, le son généré était plus étouffé et feutré. Le fidèle avait alors pleinement conscience d'être dans un lieu marqué par l'euphèmia, une ambiance sonore harmonieuse, propice au recueillement et au contact avec les dieux ; mieux, il y contribuait lui-même physiquement et devenait ainsi partie intégrante du dispositif.

Le fait de se déchausser revenait en outre à marquer une forme de déférence vis-à-vis de la divinité et participait du dépouillement recherché. Le corps se trouvait ainsi libéré des entraves habituelles, accédant à une plus grande liberté de mouvement. Cela pouvait faciliter l'exécution de performances orchestriques ${ }^{77}$ - or on sait que les rites pratiqués à Lykosoura comprenaient précisément des danses ${ }^{78}$. On constate que la suite de l'inscription proscrit indirectement (et seulement pour les initiés ?) les coiffures tressées et le port du voile : la tête devait rester visible, les cheveux dénoués. L'interdiction des bandeaux ceignant les chevelures est généralement comprise, comme pour les sandales, pourvues de lanières, de manière symbolique : il s'agissait d'éviter nœuds et ligatures, associés aux pratiques magiques d'envoûtement. Mais si l'on se place du côté des fidèles, on doit songer que le fait de laisser flotter sa chevelure et de ne pas porter de voile remonté sur la tête devait conférer un sentiment de liberté - surtout aux femmes dont le corps était généralement enserré et limité dans ses mouvements, à la différence de celui de l'homme habitué aux exercices gymniques effectués nus. Si des danses étaient effectivement pratiquées, le fait d'avoir la chevelure déliée jouait un rôle encore plus grand - comme dans la procession décrite par Callimaque, dans son Hymne à Déméter, où les femmes vont toutes pieds nus et cheveux lâchés ${ }^{79}$.

Dernier élément prohibé dans l'inscription : les fleurs. On ignore malheureusement dans

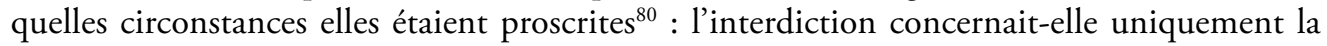
cérémonie de l'initiation ? La mesure, quoi qu'il en soit, ne manque pas de surprendre. Le règlement d'Andanie, pourtant très détaillé, ne comporte en effet aucune référence analogue. De plus, on sait que les guirlandes et couronnes végétales ou fleuries faisaient partie intégrante des rituels, diffusant leurs subtiles fragrances, ornant les autels. L'ensemble des participants étaient généralement couronnés ; à Andanie, par exemple, on utilisait du laurier.

76 Helmann 2006, 208 : «Durant toute l'époque grecque, les voies sacrées sont restées ordinairement privées de dalles".

77 L'idée m’a été suggérée par Agnès Kedzierska Manzon qui a étudié les cultes de possession en Afrique. Elle a noté que les fidèles entrant dans le périmètre de l'espace rituel se déchaussent. L'une des raisons de cette règle est pratique : les pas de danse effectués dans le cadre de ces cultes sont complexes et l'abandon des chaussures par les femmes (surtout si elles sont à talons) offre une plus grande liberté de mouvement.

78 Voir Jost 2008, 107. L'auteur suggère des danses masquées, effectuées au son de l'aulos et de la cithare : l'effet contribuait au climat "inspiré » et " enthousiasmant " qui faisait l'originalité du culte arcadien. Marie-Hélène Delavaud-Roux signale l'existence de danses orchestiques pour Déméter - en particulier lors des Thesmophories à Athènes, mais nous n'en savons guère plus (Delavaud-Roux 1994, 31-32).

79 Callimaque, Hymne à Déméter, 124-125.

80 F. Sokolowski invite à établir un parallèle avec une inscription d'Aigialè, concernant la fondation de Critolaos, qui mentionne des fleurs (LSS 61, 61). 
Comme la suite de l'inscription, relative aux offrandes, autorise la consécration de myrte et de pavot blanc, il est possible que la restriction ait été limitée à la célébration des Mystères. Si c'était le cas, la mesure contribuait à singulariser ce temps-là dans le calendrier de l'année, du point de vue visuel et olfactif : un renoncement temporaire à l'une des sources de plaisir sensuel habituelles, qui relevait, comme d'autres accessoires prohibés, de la sphère de la séduction féminine.

\section{Conclusion : éprouver ensemble et se sentir plus proche de la divinité}

Même s'il est difficile de composer avec les formulations laconiques adoptées dans les inscriptions, on dispose d'indices suffisants pour affirmer que les aspects sensoriels n'étaient pas de simples « accessoires » de mise en scène, mais des ingrédients à part entière du dispositif rituel. D’une part, ils créaient les conditions nécessaires à son efficacité, en agissant sur les dévots, leur comportement et leur état affectif, et d'autre part, ils participaient à la construction de la puissance divine. J'aimerais, pour finir, revenir successivement sur ces deux points, à la lumière des exemples que nous avons étudiés.

Les interdits promulgués avaient deux principaux types d'effets sur les fidèles. Le premier : les mettre en condition et accroître leur disponibilité intérieure et leur réceptivité aux stimuli sensoriels caractérisant l'espace sacré. Ce préalable était nécessaire pour accueillir la plénitude de la présence divine. En pénétrant dans le sanctuaire, ou lors de la procession, les fidèles éprouvaient alors une forme de dépaysement sensoriel, conditionné par les règles auxquelles ils avaient dû se soumettre, et qui les prédisposaient à éprouver un état émotionnel spécifique. Dans le cas des fêtes en l'honneur de Déméter, les femmes approchaient de l'émotion prêtée à la divinité dans l'Hymne homérique à Déméter ou dans les mythes locaux. Deuxième effet : créer une communauté de fidèles unie par une expérience sensorielle et émotionnelle partagée et contrôlée. Les mesures restrictives concouraient en effet à renforcer le sentiment de faire partie d'un même groupe, à un moment précis et dans un lieu donné. Il s'agissait non seulement d'établir les conditions nécessaires à une expérience singulière vécue par la/le participant, mais aussi de constituer une communauté réunie autour d'un partage des émotions, même si les degrés d'implication des unes et des autres devaient différer.

Les subtiles différences observées dans les trois cas étudiés révèlent en outre que chaque sanctuaire veillait à afficher ses spécificités cultuelles en se distinguant soigneusement des autres sanctuaires, jouant sur une dialectique familiarité/singularité. La configuration cultuelle variait en fonction des compétences de la divinité locale. S'il est difficile de mesurer l'impact de l'interdit du manteau décoré, pour la Déméter Thesmophore d'Achaïe, on constate qu’à Dymé et à Lykosoura, le feuilletage d'objets prohibés fournissait aux fidèles une bonne idée de l'éventail des compétences des déesses titulaires du lieu. Pour mesurer la part d'originalité de chacun de ces cultes, il suffira de rappeler qu'il existait des traditions arcadiennes mobilisant d'autres facettes de Déméter. Toutes les fêtes qui lui étaient consacrées ne requéraient pas le dépouillement attendu à Dymé ou en Achaïe : à Basilis, par exemple, des concours de beauté étaient organisés en l'honneur de Déméter Eleusinia et les femmes avaient alors justement le droit d'arborer des bijoux et ornements d'or sur leurs vêtements ${ }^{81}$.

Pour terminer, revenons sur les trois notions que nous avions évoquées en début d'article : 
polysensorialité, hypersensorialité et hyposensorialité. Nos exemples révèlent qu'en réalité, les configurations sensorielles ne sont pas aussi tranchées. Ainsi, les proscriptions relatives aux objets susceptibles de stimuler le sens de la vue, de l'ouïe, de l'odorat, permettaient en fait, en retour, d'exacerber d'autres types de sensations. C'est donc la volonté de modifier les modes d'attention qui fournit une des clefs de compréhension des interdits considérés. Ce que l'on pourrait prendre pour une recherche d'hyposensorialité relevait en fait, tout autant, de l'hypersensorialité (par exemple, sentir le contact avec le sol quand on enlève ses sandales). Il me semble donc qu'en adoptant le point de vue des autorités à l'origine des normes rituelles, les recherches actuelles ont eu tendance à considérer tous les interdits comme autant de mesures coercitives, relevant de la législation somptuaire. Or, si l'on tourne plutôt notre regard vers les participant(e)s au rituel, on constate qu'un certain nombre de "contraintes " imposées par la réglementation induisaient de fait une forme de liberté.

Adeline Grand-Clément Université de Toulouse Jean Jaurès /IUF PLH-Erasme, Maison de la Recherche 5 allées Antonio Machado 31058 Toulouse Cedex 9

France adelinegc@yahoo.fr

\section{Abréviations}

$C G R N=$ J.-M. Carbon, S. Peels and V. Pirenne-Delforge, A Collection of Greek Ritual Norms (CGRN), Liège 2016- (http://cgrn.ulg.ac.be, consulted in 2017).

$L S A M=$ F. SoKolowski, Lois sacrées de l'Asie Mineure, Paris, 1955.

LSS $=$ id., Lois sacrées des cités grecques, Supplément, Paris, 1962.

$L S C G=i d .$, Lois sacrées des cités grecques, Paris, 1969.

$N G S L=$ E. Lupu, Greek Sacred Law: A Collection of New Documents, Leiden/Boston, 2009. 


\section{Bibliographie}

Beattie 1947

A.J. Beattie, "Notes on an Arcadian Inscription concerning Demeter Thesmophoros ", The Classical Quarterly 41 (1947), 66-72.

BÉLIS 2011

A. Bélis, "Esthétique musicale du péan à travers l'exemple des Hymnes delphiques à Apollon ", in P. Brulé et Chr. Vendries (dir.), Chanter les dieux.Musique et religion dans l'Antiquité grecque et romaine, Rennes 2001, 97-114.

Bielman 2002

A. Bielman Sanchez, Femmes en public dans le monde hellénistique, $I V^{E}-I^{e r}$ siècles av. J.-C., Paris 2002.

BRULÉ 2012

P. Brulé, Comment percevoir le sanctuaire grec? Une analyse sensorielle du paysage sacré, Paris 2012.

Carbon et Pirenne-Delforge 2012

J.-M. Carbon et V. Pirenne-Delforge, «Beyond Greek "Sacred Laws" ", Kernos 25 (2012), 163 182.

Chaniotis 2010

A. Chaniotis, «The Molpoi Inscription: Ritual Prescription or Riddle? ", Kernos 23 (2010), 375-379.

\section{Cleland 2017}

L. Cleland, "A Hierarchy of Women: Status, Dress and Social Construction at Andania ", in M.-L. Nosch et C. Brons (éd.), Textiles and Cult in the Ancient Mediterranean, Oxbow 2017.

\section{Culham 1986}

P. Culham, "Again, What Meaning Lies in Colour! ", Zeitschrift für Papyrologie und Epigraphik 64 (1986), 235-245.

Delavaud-Roux 1994

M.-H. Delavaud-Roux, Les danses pacifiques en Grèce antique, Aix-en-Povence 1994.

Delli Pizzi 2011

A. Delli Pizzi, "Impiety in Epigraphic Evidence ", Kernos 24 (2011), 59-76.
Deshours 2006

N. Deshours, Les mystères d'Andania, Paris 2006.

FrISONE 2000

F. Frisone, Leggi e regolamenti funerari nel mondo Greco. I. Le fonti epigrafiche, Lecce 2000.

GAWLINSKI 2012

L. Gawlinski, The Sacred Law of Andania: A New Text with Commentary, Berlin 2012.

Georgoudi 2011

S. Georgoudi, "La procession chantante des Molpoi de Milet ", in P. Brulé, Chr. Vendries (dir.), Chanter les dieux. Musique et religion dans l'Antiquité grecque et romaine, Rennes 2001, 153-172.

\section{Georgoudi 2014}

S. Georgoudi, "Chruseos kosmos. L'usage ambivalent de l'or dans les pratiques rituelles grecques ", in M. Tortorelli Ghidini (éd.), $A u$ rum. Funzioni e simbologie dell'oro nelle culture del Mediterraneo antico, Rome 2014, 249-275.

Gherchanoc 2011

F. Gherchanoc, "Maquillage et identité : du visage au masque, de la parure à l'artifice, de la décence à l'outrage ", in L. Bodiou, F. Gherchanoc, V. Huet, V. Mehl (éd.), Parures et artifices : le corps exposé dans l'Antiquité, Paris 2011, 23-44.

Grand-Clément 2011

A. Grand-Clément, "Du bon usage du vêtement bariolé en Grèce ancienne ", in L. Bodiou, F. Gherchanoc, V. Huet et V. Mehl (éd.), Parures et Artifices: le corps exposé dans l'antiquité gréco-romaine, Paris 2011, 255-273.

Grand-ClÉment 2016a

A. Grand-Clément, "Couleurs, rituels et normes religieuses en Grèce ancienne ", Archives de Sciences sociales des religions 174 (2016), 127-147.

Grand-Clément 2016b

A. Grand-Clément, " Gold and Purple: Brilliance, Materiality and Agency of Color in Ancient Greece ", in R. Goldman (ed.), Essays in Global Color History: Interpreting the Ancient Spectrum, Piscataway 2016, 121-137. 
Helmann 2006

M.-Chr. Helmann, L'architecture grecque, tome 2: L'architecture religieuse et funéraire, Paris 2006.

JEFFERY 1949

L. H. Jeffery, "Comments on some Greek Archaic Inscriptions ", JHS 69 (1949), 30-31.

Jones 1999

Chr. Jones, "Processional Colors ", Studies in the History of Art 56 (1999), 246-257.

Jost 1985

M. Jost, Sanctuaires et cultes d'Arcadie, Paris 1985.

Jost 2008

M. Jost, « La vie religieuse à Lykosoura ", Ktèma 33 (2008), 93-110.

Karatas 2013

A.-M. Karatas, «Dress-codes related to the cult of Demeter and to other Greek cults», in Textiles and Cult in the Mediterranean Area in the first Millennium BC, International Workshop Copenhagen, 21t and 22nd of November 2013.

LANÉRÈs 2012

N. Lanérès, "La notion d'agalma dans l'épigraphie grecque », Mètis 10 (2012), 137-173.

Loucas, Loucas-Durie 1985-1986

I. Loucas, E. Loucas-Durie, " La mention du mot agalma dans la loi sacrée de Lykosoura $I G \mathrm{~V}$ 2, 514 1. 15 ", Peloponnesiaka 16 (19851986), 561-578.

Loucas, Loucas 1994

I., E. Loucas, "The Sacred Laws of Lykosoura ", in R. Hägg (ed.), Ancient Greek cult practice form the epigraphical evidence, Stockholm 1994, 97-99.

MiLls 1984

H. Mills « Greek Clothing Regulation: Sacred and Profane ", ZPE 55 (1984), 255-265.

Montel 2008

S. Montel, «Des écrins architecturaux sacralisants ? Regards sur la présentation de quelques groupes statuaires du monde grec ", in S. Estienne et alii (éd.), Image et religion dans l'Antiquité gréco-romaine, Naples 2008, 165-179.
Morizot 2004

Y. Morizot, 2004, "Offrandes à Artémis pour une naissance. Autour du relief d'Achinos ", in V. Dasen (éd.), Naissance et petite enfance dans l'Antiquité, Fribourg 2004, 159-170.

NeILs 2009

J. Neils, "Textile Dedications to Female Deities : The Case of the Peplos ", in C. Prêtre (éd.), Le donateur, l'offrande et la déesse, Liège 2009, 135-147.

Parker 1983

R. Parker, Miasma: Pollution and Purification in Early Greek Religion, Oxford 1983.

Parker 2004

R. Parker, "What are sacred laws ?", in E. Harris et L. Rubinstein (ed.), The Law and the Courts in Ancient Greece, Londres 2004, 57-70.

Peels 2016

Saskia Peels, Hosios. A Semantic Study of Greek Piety, Leiden-Boston 2016.

Piettre 2001

Piettre R., «Images et perception de la présence divine en Grèce ancienne ", Mélanges de l'École française de Rome 113 (2001), 211-224.

Piolot 2001

L. Piolot, « Le recrutement des musiciens pour les fêtes à l'époque hellénistique : le cas messénien ", in P. Brulé et Chr. Vendries (dir.), Chanter les dieux. Musique et religion dans l'Antiquité grecque et romaine, Rennes 2001, 279-306.

Platt 2011

V. Platt, Facing the Gods. Epiphany and representation in Graeco-Roman Art, Literature and Religion, Cambridge 2011.

Pugliese Carratelli 1957

G. Pugliese Carratelli, « Epigrafi del demo Coo di Isthmos ", La parola del passato (1957), 442447.

RIZAKIS 2008

A. Rizakis, Achaïe III. Les inscriptions des cités achéennes. Épigraphie et histoire, Athènes 2008.

RoBinson 1943

D.M. Robinson, "A New Arcadian Inscription ", Classical Philology 38 (1943), 191-199. 
A. Grand-Clément, "Il est interdit de... ». Rituels et procédures de régulation sensorielle dans le monde grec ancien

\section{SмIтн 1979}

P. Smith, «L'Efficacité des interdits ", L'Homme 19, 1 (1979), 5-47.

\section{STALLSMith 2008}

A.B. Stallsmith, "The name of Demeter Thesmophoros », GRBS 48 (2008), 115-131.

\section{UsTinOVA 2013}

Y. Ustinova, "To live with Joy and die with Hope: Experiential Aspects of Ancient Greek Mystery Rites ", BICS 56 (2013), 105-123. 